\title{
Mathematical models of the interrelated dynamics of hepatitis D and B
}

\author{
Aaron Packer ${ }^{\mathrm{a}, *}$, Jonathan Forde ${ }^{\mathrm{b}}$, Sarah Hews ${ }^{\mathrm{c}}$, Yang Kuang ${ }^{\mathrm{a}, \mathrm{d}}$ \\ ${ }^{a}$ School of Mathematical and Statistical Sciences, Arizona State University, Tempe, AZ 85287, USA \\ ${ }^{\mathrm{b}}$ Department of Mathematics and Computer Science, Hobart and William Smith Colleges, Geneva, NY 14456, USA

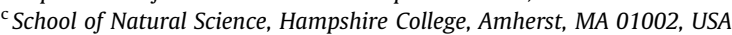 \\ ${ }^{\mathrm{d}}$ Department of Mathematics, King Abdulaziz University, Jeddah 21589, Saudi Arabia
}

\section{A R T I C L E I N F O}

\section{Article history:}

Received 11 January 2013

Received in revised form 3 October 2013

Accepted 14 October 2013

Available online 24 October 2013

\section{Keywords:}

Hepatitis delta

Hepatitis B

Mathematical model

Lamivudine

HBV

HDV

\begin{abstract}
A B S T R A C T
The hepatitis delta virus (HDV) is a rarest form of viral hepatitis, but has the worst outcomes for patients. It is a subviral satellite dependent on coinfection with hepatitis B (HBV) to replicate within the host liver. To date, there has been little to no modeling effort for HDV. Deriving and analyzing such a mathematical model poses difficulty as it requires the inclusion of (HBV). Here we begin with a well-studied HBV model from the literature and expand it to incorporate HDV. We investigate two models, one with and one without infected hepatocyte replication. Additionally, we consider treatment by the drug lamivudine. Comparison of model simulations with experimental results of lamivudine treatment indicate that infected cell proliferation may play a significant role in chronic HDV infection. Our results also shed light on several questions surrounding HDV and illustrate the need for more data.
\end{abstract}

(c) 2013 Elsevier Inc. All rights reserved.

\section{Introduction}

The hepatitis delta virus (HDV) is a dependent satellite virus of hepatitis B virus (HBV). While HDV is capable of copying its genome in great numbers in infected hepatocytes, it requires the hepatitis B surface antigen (HBsAg) in order to create new free virus particles $[1,2]$. Thus, in order for HDV to successfully complete its replication cycle, a hepatocyte must be coinfected with HBV and HDV. In these coinfected cells, the replication of HBV is suppressed by HDV, although not completely abolished [3,4].

There are many open questions surrounding the HBV-HDV coinfection system. There is currently no effective treatment for chronically infected patients, and it is unclear why the outcomes for patients with HDV are so much worse than those for patients with HBV alone [1,5]. Further, the measured levels of HBV and HDV can exhibit a wide range of dynamic behaviors in different patients, including synchronized and alternating oscillations [3].

The open questions surrounding hepatitis delta, along with the rich dynamics of the interacting populations of virions and hepatocytes make it an excellent subject for study with the tools of mathematical modeling. To date, there have been very few attempts in the literature to create a mathematical model of HBV-HDV dynamics. The earlier models of Zhang et al. [6,7] are related in that they consider the competing dynamics of two viruses in a host plant

\footnotetext{
* Corresponding author. Tel.: +1 6026955512.

E-mail addresses: ampacker@asu.edu (A. Packer), forde@hws.edu (J. Forde) shews@hampshire.edu (S. Hews), kuang@asu.edu (Y. Kuang).
}

population. The first known HBV-HDV model was proposed by Sousa and Cunha [8] and investigated numerically. Developing a model of HDV infection in vivo is made difficult by the relative lack of experimental data about the course of infection and the inherent complexity of the system. Minimally, a model must include compartments for uninfected, HBV-infected, HDV-infected, and coinfected hepatocytes. Additional compartments are needed for free HBV and HDV particles. In this paper, we develop a basic model for the interaction of HBV and HDV infection, conduct an analysis of its steady state structure and stability, and test model behavior against experimental results from drug treatment trials [9-11].

\section{HBV model}

Given the important role that HBV plays in HDV dynamics, we start by defining a model for infection by HBV alone. We consider three distinct population concentrations within the liver: healthy hepatocytes $(x)$, HBV infected hepatocytes $(y)$, and free HBV virions $(v)$. The dynamics are governed by the following set of assumptions:

1. Healthy cells are regenerated.

2. Healthy cells transition to an infected state due to infection by free virions.

3. Infected cells die at an increased rate due to infection.

4. Virions are produced by infected cells.

5. Virions die at a specified rate. 
The exact functions which govern these dynamics depend on further assumptions. This general framework stems from simple, linear models [12] which have been refined and improved upon over time, as there are many rich HBV models in the literature [1317]. For an initial HDV model, we begin with the ODE model proposed in Hews et al. [18], which produces rich and biologically meaningful dynamics by replacing the traditional mass action infection terms with nonlinear but more realistic rate functions [13].

$\frac{d x}{d t}=r x(t)\left(1-\frac{T(t)}{K}\right)-\frac{\beta v(t) x(t)}{T(t)}$,

$\frac{d y}{d t}=\frac{\beta v(t) x(t)}{T(t)}-a y(t)$

$\frac{d v}{d t}=\gamma y(t)-\mu v(t)$

with

$T(t)=x(t)+y(t)$.

The uninfected hepatocyte population is assumed to maintain itself logistically [16], with a homeostatic carrying capacity of $K$. The infection rate is proportional to the incidence function $\beta v(t) x(t) / T(t)$. This replaces the mass action function [12], which has been shown to cause unrealistic conditions for successful chronic HBV infection $[13,17]$. The ratio-dependence incidence function is more general and is mathematically equivalent to mass action when the total population is constant. The production of virions and the death of virions and infected cells occur at constant per capita rates.

\subsection{Reduced HBV model}

Incorporating HDV infection into Eqs. (1)-(3) will require three more populations: HDV infected cells, HBV-HDV coinfected cells, and free HDV virions. In order to reduce the number of equations and derive a more mathematically tractable HDV model, the HBV model is first simplified into a planar system. Since the time scale of virion production is much shorter than the time scale of the hepatocytes life-cycle, we assume that the free virus population is in quasi-steady-state with the infected cell population. Thus, we set $\frac{d v}{d t}=0$ and conclude that $v$ is proportional to $y\left(v=\frac{\gamma}{\mu} y\right)$, giving the following "reduced" HBV model:

$\frac{d x}{d t}=r x(t)\left(1-\frac{T(t)}{K}\right)-\frac{\sigma y(t) x(t)}{T(t)}$,

$\frac{d y}{d t}=\frac{\sigma y(t) x(t)}{T(t)}-a y(t)$

where

$\sigma=\beta \gamma / \mu$.

Mathematically, the reduced model exhibits similar dynamics as the original for biologically relevant parameter values. They share the same basic reproduction number,

$R_{0}=\frac{\beta \gamma}{\mu a}=\frac{\sigma}{a}$

and the coordinates of the steady states are unchanged. It is easy to verify that they have biologically equivalent steady states, with the reduced model having $E_{0}=(0,0), E_{f}=(K, 0)$, and $E^{*}=\left(x^{*}, y^{*}\right)$, where
$x^{*}=\frac{K a}{r}\left(\frac{r+a}{a R_{0}}-1\right)$,

$y^{*}=\left(R_{0}-1\right) x^{*}$.

Thorough analyses for the original and reduced models are available in Hews et al. [18] and Hwang and Kuang [19,20], respectively. Asymptotically the models are similar, except the planar system loses a region in which solutions approach stable limit cycles. In both systems, $E_{f}$ is globally asymptotically stable for $R_{0}<1$ and there is a stability switch to $E^{*}$ at the bifurcation point $R_{0}=1$. Increasing $R_{0}$ leads to stability of $E_{0}$ in both systems at a specific point $R_{0}>1$. However, as $R_{0}$ increases towards this value, the original system first enters a region of stable periodic orbits. See Fig. 1 for an illustrative example of both models using the same parameter values.

Despite this dissimilarity, both models yield essentially the same biological insight: if viral reproduction is too aggressive relative to the liver's regenerative ability, then complete liver failure can result with both the healthy cells and virus population becoming extinct. These properties suggest that for successful chronic infection, the virus and liver must coexist with a balance which is governed by $R_{0}$ being neither too large nor too small.

\section{HDV model}

In order to incorporate HDV into Eqs. (5) and (6), we add two further state variables, accounting for hepatocytes infected with HDV and those coinfected with both HBV and HDV. The state variables in the expanded model are

1. $x(t)=$ uninfected cells,

2. $y(t)=$ HBV only infected cells,

3. $z(t)=$ HDV only infected cells,

4. $w(t)=$ coinfected cells .

The model structure is similar to other two virus systems [6,8], with the state transitions defined as the possible infection events that can occur. We assume the following transitions are possible:

1. $x \rightarrow y$ : healthy cell infected by HBV,

2. $x \rightarrow z$ : healthy cell infected by HDV,

3. $y \rightarrow w$ : HBV infected cell superinfected by HDV,

4. $z \rightarrow w$ : HDV infected cell superinfected by HBV.

The rates at which these transitions take place are proportional to the viral concentrations. As before, we assume that the free virus populations are in a quasi-steady-state relation with the infected cell populations. As free HDV particles can only be produced by coinfected cells, the number of HDV virions is proportional to the number of coinfected cells, $w$. HBV virions are produced by hepatocytes infected with HBV alone ( $y$ ) and also by coinfected hepatocytes $(w)$, although at a reduced rate in the latter cells. Letting $c$ be the degree to which HBV production is suppressed in coinfected cells, we conclude that the number of HBV virions is proportional to $y+c w$. Using the same form of incidence functions as in the HBV model we arrive at the following system of differential equations.

$$
\begin{aligned}
& \frac{d x}{d t}=r x(t)\left(1-\frac{T(t)}{K}\right)-\frac{\sigma(y(t)+c w(t)) x(t)}{T(t)}-\frac{\delta w(t) x(t)}{T(t)}, \\
& \frac{d y}{d t}=\frac{\sigma(y(t)+c w(t)) x(t)}{T(t)}-\frac{\delta w(t) y(t)}{T(t)}-a y(t), \\
& \frac{d z}{d t}=\frac{\delta w(t) x(t)}{T(t)}-\frac{\sigma(y(t)+c w(t)) z(t)}{T(t)}-a z(t),
\end{aligned}
$$




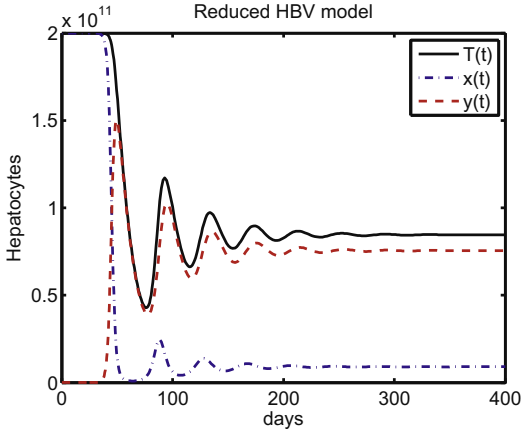

(a)

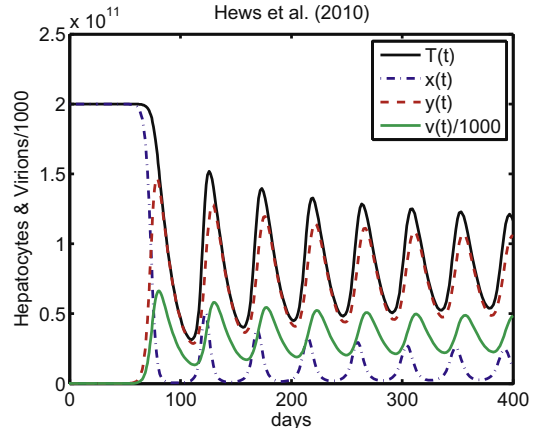

(b)

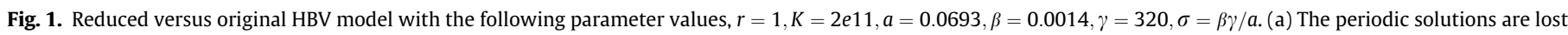
in the reduced model. (b) The original HBV model contains stable periodic orbits.

$\frac{d w}{d t}=\frac{\sigma(y(t)+c w(t)) z(t)}{T(t)}+\frac{\delta w(t) y(t)}{T(t)}-a w(t)$,

where

$T(t)=x(t)+y(t)+z(t)+w(t)$.

Refer to Table (1) for a complete list of parameter descriptions and units.

\subsection{Model properties}

For the following, observe that

$\frac{d T}{d t}=r x(t)(1-T(t) / K)-a(y(t)+z(t)+w(t))$.

Proposition 1. Solutions to Eqs. (8)-(11) with strictly positive initial conditions and $K \geqslant T(0)=x(0)+y(0)+z(0)+w(0)$ remain strictly positive and bounded for all time. Further, $T(t) \leqslant K$ for all $t \geqslant 0$.

Proof. Let $(x(t), y(t), z(t), w(t))$ be a solution to Eqs. (8)-(11) with positive initial conditions and $K \geqslant T(0)=x(0)+y(0)+$ $z(0)+w(0)$. Suppose there exists a $t_{1}>0$ such that $x(t)>0, y(t)>0, z(t)>0, w(t)>0$ for $t \in\left[0, t_{1}\right)$, but that one or multiple solution components are zero at $t_{1}$. Observe that for $t \in\left[0, t_{1}\right],-a T(t) \leqslant T^{\prime}(t) \leqslant r T(t)(1-T(t) / K)$, which implies by a simple comparison argument that $0<T(t) \leqslant K$. So for $t \in\left[0, t_{1}\right], x(t) \leqslant K, y(t) \leqslant K, z(t) \leqslant K, w(t) \leqslant K$. Consider now four possible (but not mutually exclusive) cases:

First, that $w\left(t_{1}\right)=0$. But from Eq. (11) we have that $w^{\prime}(t) \geqslant-a w(t)$, which implies $w(t) \geqslant w(0) e^{-a t}>0$, a contradiction. Hence $w(t)>0$ for all $t$.

Second, that $y\left(t_{1}\right)=0$. But $0 \leqslant w(t) \leqslant K$ implies $0<w(t) / T(t) \leqslant 1$. Then Eq. (9) yields $y^{\prime}(t) \geqslant-(\delta+a) y(t)$. Then $y(t) \geqslant y(0) e^{-(\delta+a) t}>0$, a contradiction. Hence $y(t)>0$ for all $t$.

Table 1

Parameter descriptions.

\begin{tabular}{lll}
\hline Parameter & Description & Units \\
\hline$r$ & Maximum proliferation rate & day $^{-1}$ \\
$K$ & Homeostatic liver size & cells \\
$a$ & Infected cell death rate & day $^{-1}$ \\
$c$ & HBV inhibition coefficient & scalar \\
$\beta$ & HBV infection rate & day $^{-1}$ \\
$\gamma$ & HBV replication/release rate & day $^{-1}$ \\
$\mu$ & Hepatitis B virion death rate & day $^{-1}$ \\
$\sigma$ & HBV infection rate $(\gamma \beta / \mu)$ & day $^{-1}$ \\
$\delta$
\end{tabular}

Third, that $x\left(t_{1}\right)=0$. Similarly, we obtain a contradiction. So $x(t)>0$ for all $t$.

Fourth, that $z\left(t_{1}\right)=0$. Similarly, we obtain a contradiction. So $z(t)>0$ for all $t$.

\subsection{Steady states}

System (8)-(11) has four steady states, three of which are analogous to those of the reduced HBV model (5) and (6): $E_{0}=(0,0,0,0), E_{f}=(K, 0,0,0)$, and $E_{b}=\left(x_{b}, y_{b}, 0,0\right)$, where

$x_{b}=\frac{K a}{r}\left(\frac{r+a}{a R_{0}}-1\right)=\frac{K}{r R_{0}}(r-\sigma+a)$

$y_{b}=\left(R_{0}-1\right) x_{b}$

and as before $R_{0}=\frac{\beta \gamma}{\mu a}=\frac{\sigma}{a}$.

These three correspond respectively to complete liver failure, complete disease clearance, and HBV-only persistence. The fourth steady state $E^{*}=\left(x^{*}>0, y^{*}>0, z^{*}>0, w^{*}>0\right)$ corresponds to chronic coinfection by HBV and HDV. While the coordinates of the fourth steady state cannot be expressed explicitly, its existence is verified numerically. Note that there are no steady states in which only HDV persists. This is an expected outcome of the model formulation, since HDV depends on HBV for its replication, and the infected cell populations do not proliferate. See Fig. 2 for example solution profiles.

Fig. 3 contains bifurcation diagrams which illustrate the overall asymptotic behavior of the model with respect to $R_{b}=\sigma / a$ and $R_{d}=\delta / a$. As we show, these two values are useful for determining the overall dynamics of the system. $R_{b}>1$ is a necessary condition for stability of both $E_{b}$ and $E^{*}$, whereas $R_{d}>1$ is necessary only for $E^{*}$. We show these properties and other results below.

Proposition 2. The disease-free equilibrium $E_{f}=(K, 0,0,0)$ is locally asymptotically stable if and only if $\sigma / a<1$.

Proof. At $E_{f}$ the Jacobian is

$J=\left(\begin{array}{llll}-r & -r-\sigma & -r & -r-\delta-c \sigma \\ 0 & \sigma-a & 0 & c \sigma \\ 0 & 0 & -a & \delta \\ 0 & 0 & 0 & -a\end{array}\right)$,

which has eigenvalues $\{-r, \sigma-a,-a,-a\}$. All eigenvalues are strictly negative if and only if $\sigma / a<1$.

Proposition 3. If $\sigma / a<1$ then $\lim _{t \rightarrow \infty} y(t)=\lim _{t \rightarrow \infty} w(t)=$ $\lim _{t \rightarrow \infty} z(t)=0$. 


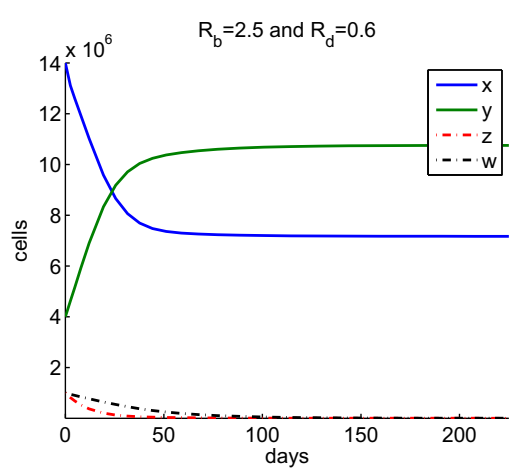

(a)

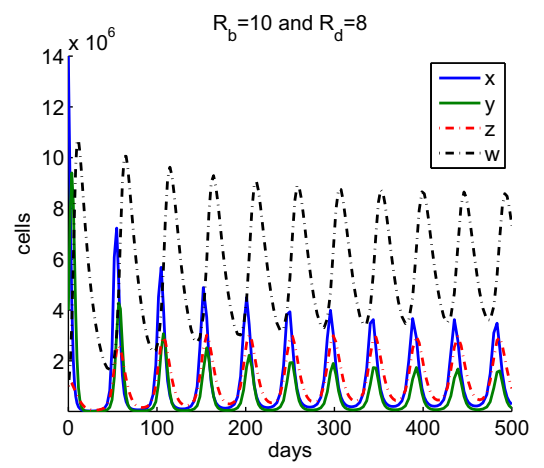

(c)

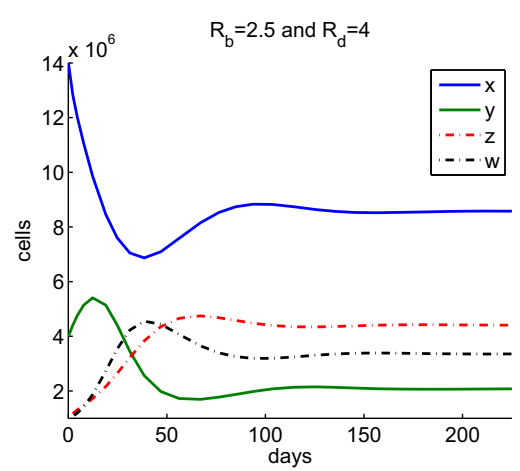

(b)

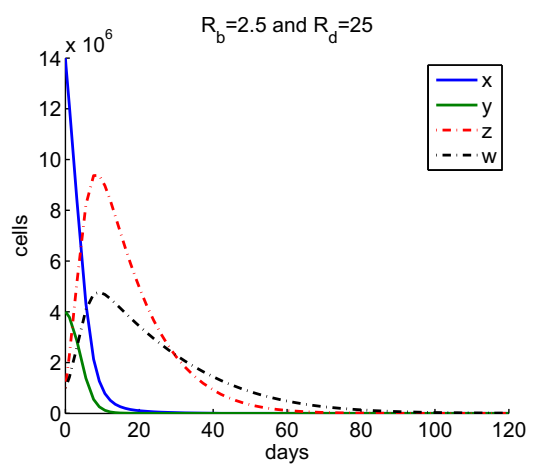

(d)

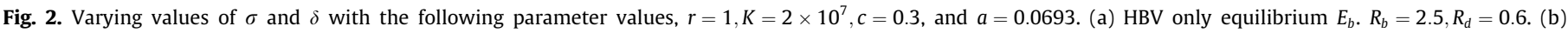

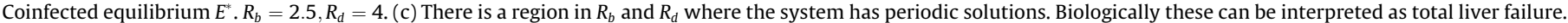
(d) High virulence leading to total liver failure, or $E_{0}$.

Proof. Suppose $\sigma / a<1$. First we show that $\lim _{t \rightarrow \infty} y(t)=$ $\lim _{t \rightarrow \infty} w(t)=0$. Since $c<1$ and $x+c z \leqslant T$, it follows that

$$
\begin{aligned}
(y+c w)^{\prime} & =\left(\frac{\sigma(x+c z)}{T}-a\right)(y+c w)-(1-c) \frac{\delta w y}{T} \\
& <(\sigma-a)(y+c w)<0
\end{aligned}
$$

which combined with the positivity of $y$ and $w$ implies the result. Now $\lim _{t \rightarrow \infty} w(t)=0$ can be used to prove that $\lim _{t \rightarrow \infty} z(t)=0$. Let $\epsilon>0$. We can choose a $t_{\epsilon}>0$ such that if $t>t_{\epsilon}$ then $w(t)<(a / \sigma) \epsilon / 2$. Further,

$$
\begin{aligned}
z^{\prime} & <\sigma w-a z \\
& <a\left(\frac{\epsilon}{2}-z\right) .
\end{aligned}
$$

Let $z_{\epsilon}(t)$ be the function defined on $\left[t_{\epsilon}, \infty\right)$ which satisfies $z_{\epsilon}\left(t_{\epsilon}\right)=z\left(t_{\epsilon}\right)$ and $z_{\epsilon}^{\prime}=a\left(\epsilon / 2-z_{\epsilon}\right)$. By a simple comparison argument, $z<z_{\epsilon}$ on $\left[t_{\epsilon}, \infty\right)$. Since $\lim _{t \rightarrow \infty} z_{\epsilon}(t)=\epsilon / 2$, there is a $t^{*}>t_{\epsilon}$ such that for all $t>t^{*}, z_{\epsilon}(t)<\epsilon$. So $z(t)<\epsilon$. Positivity of $z$ and taking $\epsilon \rightarrow 0$ yields $\lim _{t \rightarrow \infty} z(t)=0$.

Proposition ( 3 ) shows that HBV persistence is necessary for that of HDV. If $R_{b}<1$ then the value of $\delta$ has no bearing on $w$ or $z$, which monotonically decrease towards zero. Similarly, if $\delta / a<1$ then $w$ and $z$ approach zero regardless if $R_{b}>1$.

Proposition 4. If $\delta / a<1$, then $\lim _{t \rightarrow \infty} w(t)=\lim _{t \rightarrow \infty} z(t)=0$.

Proof. Assume $\delta / a<1$. Then

$$
\begin{aligned}
(w+z)^{\prime} & =\frac{\delta w}{T}(x+y)-a(w+z) \\
& <\delta(w+z)-a(w+z) \\
& <0
\end{aligned}
$$

Since $0<w+z$ it follows that $\lim _{t \rightarrow \infty}(w+z)=0$.

Theorem 1. The disease-free equilibrium $E_{f}$ is globally asymptotically stable if $\sigma / a<1$.

Proof. From Proposition (3) it suffices to show that $x \rightarrow K$. Let $\epsilon>0$. Then there is $t_{\epsilon}>0$ such that for $t>t_{\epsilon}, w(t), z(t), y(t)<\epsilon$. Then $x^{\prime}>r x\left(1-\frac{x}{K}\right)-A \epsilon$ where $A=\sigma+c \sigma+\delta+3 r$. Let $x_{\epsilon}(t)$ be the solution to the differential equation $x_{\epsilon}^{\prime}=r x_{\epsilon}\left(1-\frac{x_{\epsilon}}{K}\right)-A \epsilon$ with $x_{\epsilon}\left(t_{\epsilon}\right)=x\left(t_{\epsilon}\right) . x_{\epsilon}$ has two steady states, $x_{+}$and $x_{-}$, which are given by

$x_{ \pm}=K \frac{1 \pm \sqrt{1-\frac{4 A \epsilon}{r K}}}{2}$.

$x_{\epsilon}^{\prime \prime}=r\left(1-\frac{2 x_{\epsilon}}{K}\right)$ is strictly negative at $x_{+}$and strictly positive at $x_{-}$. Hence $x_{\epsilon} \rightarrow x_{+}$, and from $x_{\epsilon}<x \leqslant K$ we have that $x \rightarrow K$ as $t \rightarrow \infty$.

\section{Lamivudine treatment}

Lamivudine is a nucleoside analogue reverse transcriptase inhibitor which has been approved as a treatment for chronic HBV infection. The chemical is incorporated into the viral genome via HBV polymerase, and results in chain termination, blocking 


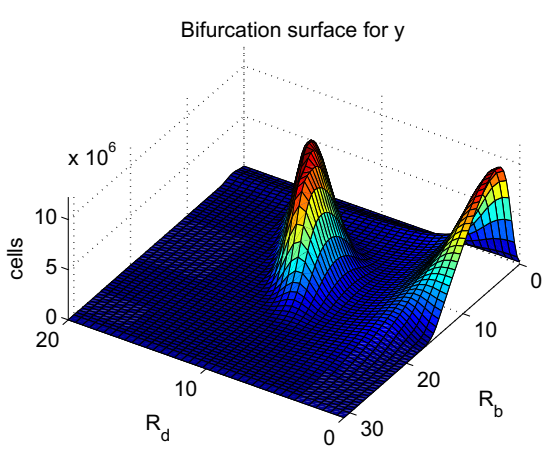

(a)

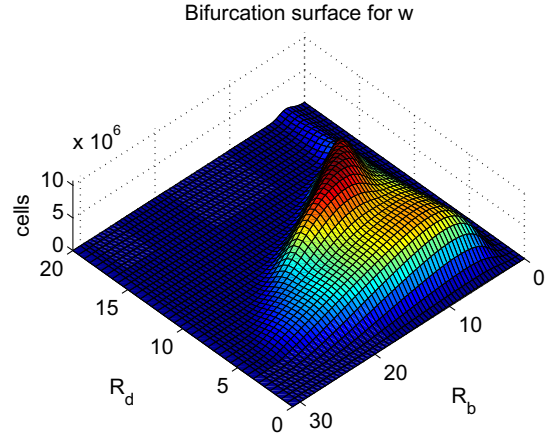

(b)

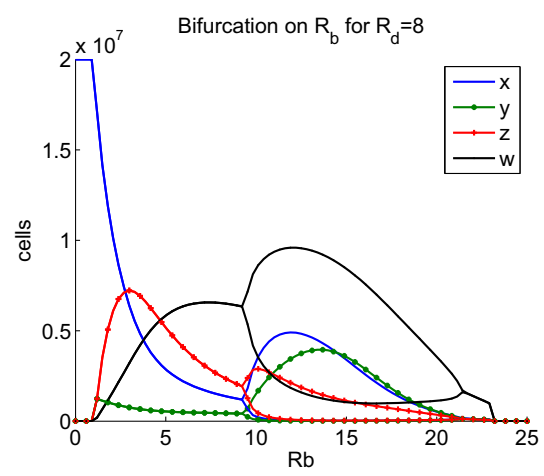

(c)

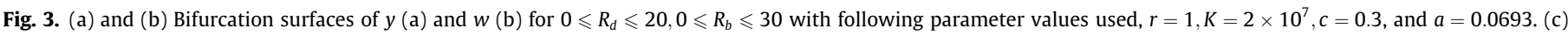
Bifurcation on $R_{b}$ for $R_{d}=8$. It illustrates the dependence of $E^{*}$ on $R_{b}$. Since $R_{d}>1$, we do not see $E_{b}$ when $R_{b}>1$.

HBV reproduction. Clinical studies have shown lamivudine to be very effective in reducing the prevalence of $\mathrm{HBV}$ in infected patients $[21,10]$. HBV DNA levels in serum are rapidly reduced, followed by a slower loss of HBsAg, which could be correlated with the loss of infected hepatocytes [22].

This drug was thus considered a worthwhile candidate of treating chronic hepatitis delta patients, since HDV is dependent on HBV. One would expect that administering lamivudine to individuals with chronic HBV and HDV would also reduce HDV levels. However, studies have shown that administering lamivudine has no significant effect on the levels of HDV RNA in infected patients $[9,11,23,10]$. Even after months of treatment, HDV viral production and liver damage (i.e. infection) remain high. Furthermore, HBV levels can return post treatment $[9,11]$. It is believed that lamivudine inhibits HBV replication downstream of RNA production, which allows for continued production of HBsAg [9].

The unique nature of these experimental results provides the opportunity to test the model of HBV-HDV infection presented in Eqs. (8)-(11). Nucleoside analogues block the production of new HBV virions. Therefore, administration of this drug will result in a reduction of the parameter $\sigma$ at all occurrences. Similar to Sousa and Cunha [8], we introduce the parameter $\eta$ to represent the efficacy of lamivudine in preventing HBV production.

\subsection{Simulation}

To simulate lamivudine treatment of patients in cases of chronic hepatitis delta infection, we add the drug efficacy parameter $\eta$ to Eqs. (8)-(11). The model with treatment is

$$
\begin{aligned}
\frac{d x}{d t}= & r x(t)\left(1-\frac{T(t)}{K}\right)-\frac{(1-\eta) \sigma(y(t)+c w(t)) x(t)}{T(t)} \\
& -\frac{\delta w(t) x(t)}{T(t)},
\end{aligned}
$$

$$
\begin{aligned}
& \frac{d y}{d t}=\frac{(1-\eta) \sigma(y(t)+c w(t)) x(t)}{T(t)}-\frac{\delta w(t) y(t)}{T(t)}-a y(t), \\
& \frac{d z}{d t}=\frac{\delta w(t) x(t)}{T(t)}-\frac{(1-\eta) \sigma(y(t)+c w(t)) z(t)}{T(t)}-a z(t), \\
& \frac{d w}{d t}=\frac{(1-\eta) \sigma(y(t)+c w(t)) z(t)}{T(t)}+\frac{\delta w(t) y(t)}{T(t)}-a w(t),
\end{aligned}
$$

where $\eta \leq 1$ during treatment and $\eta=0$ when off treatment. Treatment was induced to solutions in both chronic HBV and chronic HBV-HDV states.

\subsection{Results}

In the present model, HDV infection is sustained during treatment only if the HBV infected cell population does not become extinct. Indeed, if $(1-\eta) \sigma / a<1$, as we would expect during treatment, then the HDV and HBV-HDV infected cell populations tend to zero. If $\eta \sigma / a>1$ then the expected outcome is not possible: there is either no significant change in the solution or both HBV and HDV levels of infection decrease. Simulations were performed for the treatment model as well as an extended model with HBV and HDV free virions. Fig. 4 shows an example profile whereby both HBV and HDV infections decrease, including free virions. The model of Sousa and Cunha [8] similarly does not predict observed dynamics, with lamivudine effectively reducing both HBV and HDV viral loads.

These simulation and basic stability results indicate that the HBV-HDV coinfection system has some mechanism which sustains the population of coinfected hepatocytes even in the absence of ongoing HBV production. Perhaps the simplest way to envision 
such a mechanism is to allow for the proliferation of infected hepatocyte classes. Such a model is presented in the following section.

\section{Model with infected cell proliferation}

As with any biological model, implications are often necessary in order to gain meaningful insight into the underlying mechanics. Here we modify the original model by instead assuming that infected hepatocytes not only proliferate but also transmit infection vertically to daughter cells. The reasoning is that during treatment, HBsAg production must somehow be maintained. From the context of chronic HBV-HDV infection and lamivudine, it is reasonable to assume that infected cells should proliferate at rates sufficient to sustain infection. Empirically driven models of HBV have also included infected cell proliferation [15,24].

There is conflicting evidence on the proliferation of HBV infected hepatocytes [25], with data suggesting both inhibition $[26,27]$ and induction of proliferation [28]. However, HBV infection is indeed observed to persist across cell divisions, as infected cells often contain multiple copies of the viral genome [29]. When released into a cell, the viral genomic DNA is partially double stranded and not covalently closed. It is transported to the host nucleus and converted into covalently closed circular DNA (cccDNA) which can be used as a template for transcribing viral RNA via host enzymes. Also key to the HBV replication cycle is the intracellular amplification of cccDNA. Copies of cccDNA can be retained during cellular division or even antiviral treatment [29].

We explore a simple extension of system (8) and (9) which uses the logistic function for proliferation of both healthy and infected cells. The model is now given by

$\frac{d x}{d t}=r x(t)\left(1-\frac{T(t)}{K}\right)-\frac{\sigma(y(t)+c w(t)) x(t)}{T(t)}-\frac{\delta w(t) x(t)}{T(t)}$,

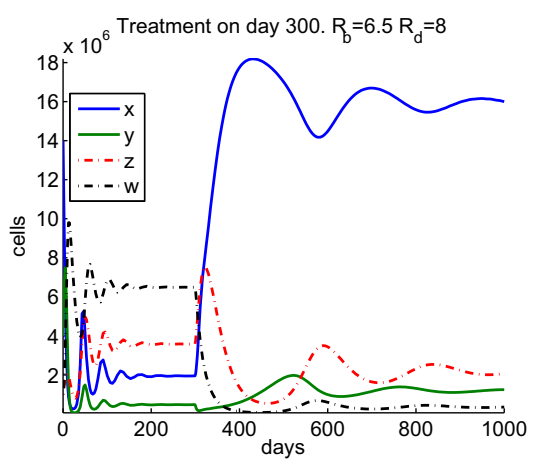

(a) $\frac{d y}{d t}=r y(t)\left(1-\frac{T(t)}{K}\right)+\frac{\sigma(y(t)+c w(t)) x(t)}{T(t)}-\frac{\delta w(t) y(t)}{T(t)}-a y(t)$,

$$
\frac{d z}{d t}=r w(t)\left(1-\frac{T(t)}{K}\right)+\frac{\delta w(t) x(t)}{T(t)}-\frac{\sigma(y(t)+c w(t)) z(t)}{T(t)}-a z(t),
$$

$$
\frac{d w}{d t}=r z(t)\left(1-\frac{T(t)}{K}\right)+\frac{\sigma(y(t)+c w(t)) z(t)}{T(t)}+\frac{\delta w(t) y(t)}{T(t)}-a w(t),
$$

with liver regeneration sustained by both healthy and infected cells, and an increased death rate for infected cells. It is assumed that infection state is always transmitted to daughter cells, including coinfection. a now captures both proliferation inhibition and increased death of infected hepatocytes. While the logistic function inherently encapsulates both "birth" and "death" processes of a population, the infected population death rate $a$ is kept as a separate term for consistency and convention. Combining $-a$ with $r\left(1-\frac{T}{K}\right)$ yields the effective "infected" logistic function, with reduced maximum rate $r-a$ and decreased carrying capacity $K(1-a / r)$ :

$(r-a)\left(1-\frac{T}{K(1-a / r)}\right)=r\left(1-\frac{T}{K}\right)-a$.

\subsection{Model properties}

System (17)-(20) has the same properties of system (8)-(11) with regards to positive invariance of the biologically meaningful domain. For this model, we similarly define $R_{b}=\sigma / a$, but now have

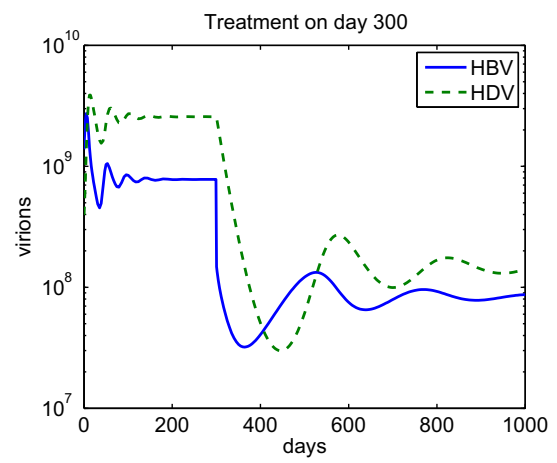

(b)

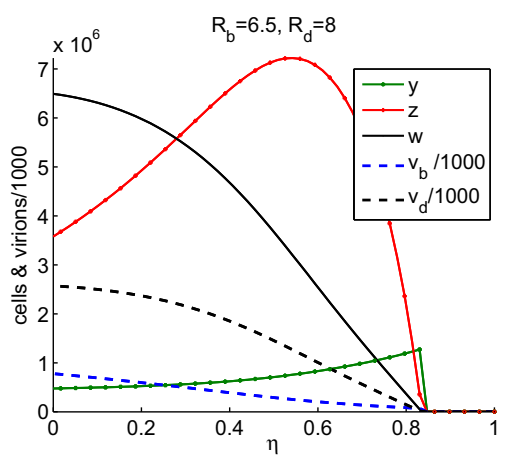

(c)

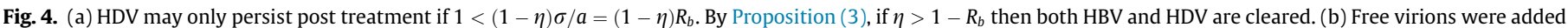

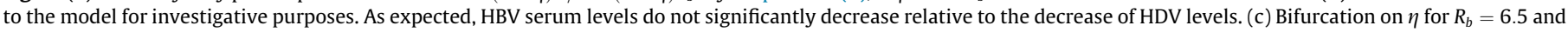
$R_{d}=8$. 


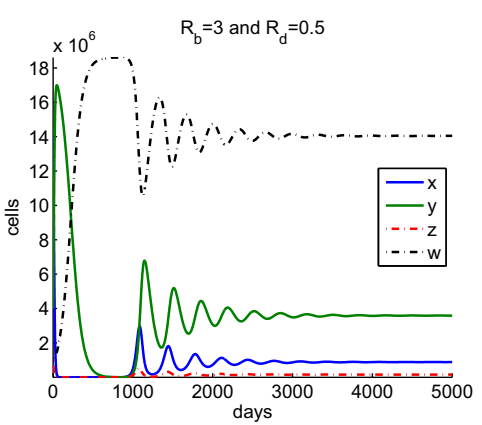

(a)

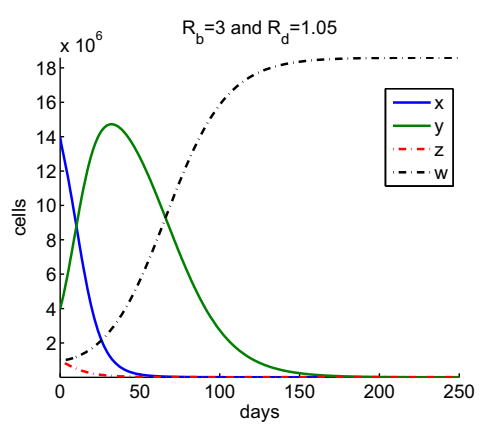

(b)

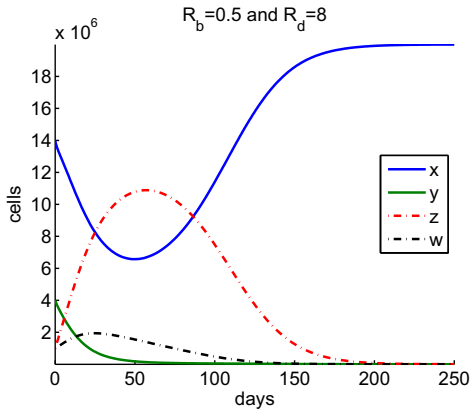

(c)

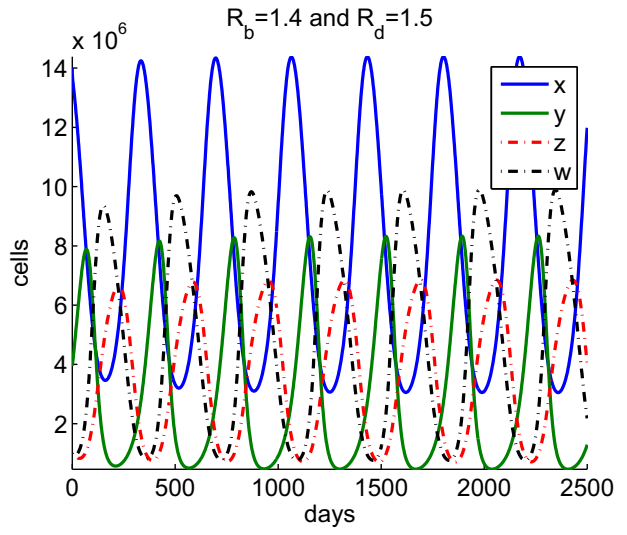

(d)

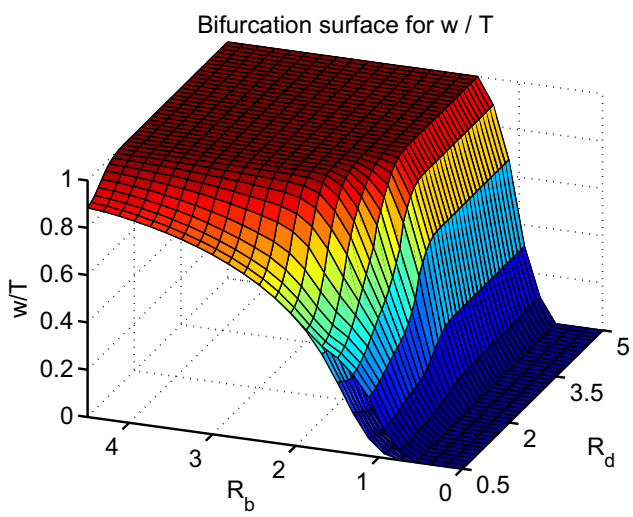

(e)

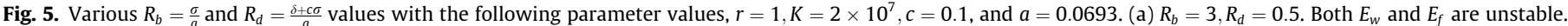

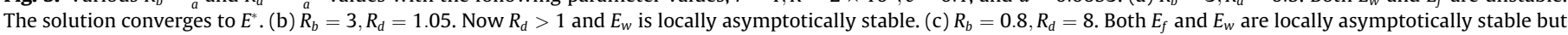

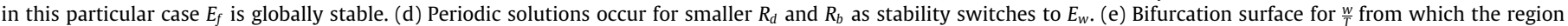
containing $(\mathrm{d})$ can be discerned.

$R_{d}=\frac{\delta+c \sigma}{a}=\frac{\delta}{a}+c R_{b}$.

Note that this creates the bound $R_{d}>c R_{b}$. The steady states of the revised model differ from those of the model without infected cell proliferation. Let $K_{1}=K\left(1-\frac{a}{r}\right)$. Recall that $K_{1}<K$ is the "infected" carrying capacity from Eq. (21), due to increased death and inhibited proliferation rates. Further, $K_{1}>0$ if and only if $a<r$, an expected condition for persistence of a deterministic population. Eqs. (17) and (18) give rise to six steady states: $E_{0}=(0,0,0,0), E_{f}=(K, 0,0,0), E_{y}=\left(0, K_{1}, 0,0\right), E_{z}=\left(0,0, K_{1}, 0\right), E_{w}$ $=\left(0,0,0, K_{1}\right)$, and $E^{*}=\left(x^{*}, y^{*}, z^{*}, w^{*}\right)$.

Deriving all steady states other than $E^{*}$ is trivial. While $E^{*}$ cannot be expressed explicitly, its existence is inferred from simulation (see Fig. 5).

Proposition 5. The disease-free equilibrium $E_{f}=(K, 0,0,0)$ is locally asymptotically stable if and only if $\sigma / a<1$.

Proof. At $E_{f}$ the Jacobian is

$J=\left(\begin{array}{cccc}-r & -r-\sigma & -r & -r-\delta-c \sigma \\ 0 & \sigma-a & 0 & c \sigma \\ 0 & 0 & -a & \delta \\ 0 & 0 & 0 & -a\end{array}\right)$,

which has eigenvalues $\{-r, \sigma-a,-a,-a\}$. All eigenvalues are strictly negative if and only if $\sigma / a<1$.
Proposition 6. The HBV-only equilibrium $E_{y}=\left(0, k\left(1-\frac{a}{r}\right), 0,0\right)$ is always unstable.

Proof. At $E_{y}$ the Jacobian is

$J=\left(\begin{array}{cccc}a-\sigma & 0 & 0 & 0 \\ a-r+\sigma & a-r & a-r & a-r-\delta \\ 0 & 0 & -\sigma & 0 \\ 0 & 0 & \sigma & \delta\end{array}\right)$,

which has eigenvalues $\{a-\sigma, a-r,-\sigma, \delta\}$. By assumption $\delta>0$.

Proposition 7. The equilibrium $E_{z}=\left(0,0, k\left(1-\frac{a}{r}\right), 0\right)$ is always unstable.

Proof. At $E_{y}$ the Jacobian is

$J=\left(\begin{array}{cccc}a & 0 & 0 & 0 \\ 0 & 0 & 0 & 0 \\ a-r & a-r-\sigma & a-r & a-r-c \sigma \\ 0 & \sigma & 0 & c \sigma\end{array}\right)$,

which has eigenvalues $\{a, 0, a-r, c \sigma\}$. By assumption $a, c \sigma>0$.

Proposition 8. The equilibrium $E_{w}=\left(0,0,0, k\left(1-\frac{a}{r}\right)\right)$ is locally asymptotically stable if and only if $a<r$ and $a<c \sigma+\delta$. 


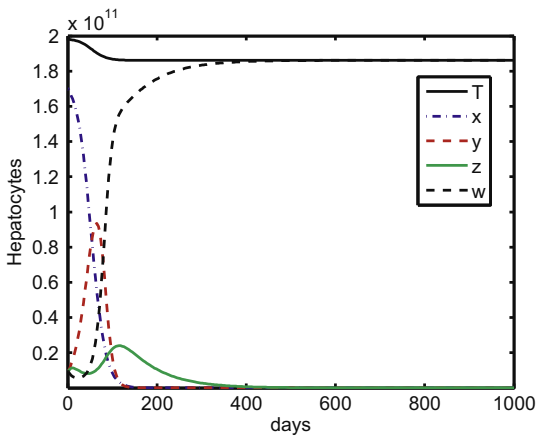

(a)

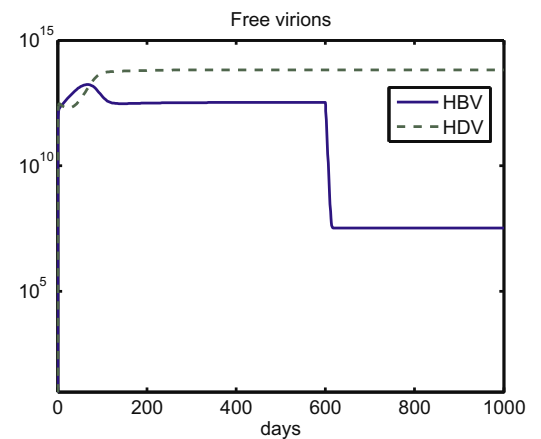

(b)

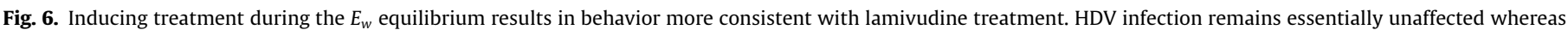

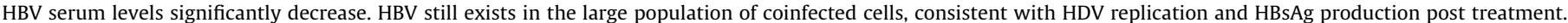

Proof. At $E_{w}$ the Jacobian is

$J=\left(\begin{array}{cccc}a-c \sigma-\delta & 0 & 0 & 0 \\ c \sigma & -\delta & 0 & 0 \\ \delta & 0 & -c \sigma & 0 \\ a-r & a-r+\delta & a-r+c \sigma & a-r\end{array}\right)$,

which has eigenvalues $\{a-c \sigma-\delta,-\delta,-c \sigma, a-r\}$. All eigenvalues are strictly negative if and only if $a<r$ and $a<c \sigma+\delta$.

Representative solutions to model (17)-(20) are found in Fig. 5. In contrast to model (8)-(11), the chronic HBV state is always unstable and there is an additional chronic coinfection state $E_{w}$. Although $R_{b}>1$ is not necessary for local stability of $E_{w}$, it is needed to reach $E_{w}$ when initial conditions are closer to $E_{f}$. Therefore both models are consistent with HDV being a subviral satellite and the intuition that adequately infectious HBV would be required to facilitate chronic infection by HDV. The two coinfection equilibria, $E^{*}$ and $E_{w}$, may represent different degrees of infection.

Treatment is added to the model in the same manner as Eqs. (13)-(16), via the drug efficacy parameter $\eta$. As the results in Fig. 6 show, the revised model exhibits the behavior observed in lamivudine treatment experiments. The reason is that stability of $E_{w}$ requires $\frac{\delta+c \sigma}{a}>1$ but not $\frac{\sigma}{a}>1$. This differs in that $(1-\eta) \frac{\sigma}{a}<1$ can only change stability of $E_{w}$ if $\frac{\delta}{a}$ is sufficiently less than one. Otherwise, when the system is treated in state $E_{w}$ the number of HDV-HBV infected cells is not affected. However, the computed HBV free virion population decreases significantly while that of HDV remains relatively stable. This decrease in HBV particles is consistent with the observed decrease in hepatitis B viral load. The HBV DNA that exists in the coinfected cells $w$ is consistent with continued HBsAg production.

If treatment is applied to the system in state $E^{*}$, the behavior is similar to that of the model without infected cell proliferation. If $(1-\eta) \sigma / a<1$ then $E_{f}$ becomes stable and, based on numerical bifurcations around $R_{b}=1, E^{*}$ becomes unstable. This suggests that $E^{*}$ represents chronic coinfection from a hepatitis D virus strain that is less infectious than a strain that would cause $E_{w}$ in the same individual.

While these results make it seem likely that infected cell proliferation plays a significant role in the dynamics of HBV-HDV coinfection, direct experimental observation of infected cell proliferation that is also a predictor of observed HDV viral load and replication would still be desirable.

\section{Discussion and conclusion}

There is still much to be learned and discovered about HDV. It is a unique virus for which many open questions remain. For example, HDV-specific treatment protocols still do not exist. The investigation of the inter-related dynamics of chronic HBV and HDV infections are important to understanding how treatment may affect this complex system. To this end, the development of biologically realistic mathematical models is an important tool.

By incorporating known biology and comparing model simulations with the results of experimental drug therapy trials, we have developed and validated a basic model for the interaction of infected hepatocyte populations in establishing chronic HDV infections. In particular, we have discovered that the observed dynamics of viral titers in lamivudine treatment can only be explained by the existence of some mechanism for the maintenance of the coinfected hepatocyte population in the absence of ongoing HBV production. This suggests that the possibility that infected hepatocytes are capable of proliferation, and pass their viral infections on the their daughter cells.

As more information becomes available, this model can be refined and expanded to incorporated more of the known biology. For example, the role of the immune reaction in controlling the extent of infection and in mediating patient symptoms is still unclear. This mathematical model provides a platform to test potential mechanisms of drug action and treatment strategies without the need to conduct costly experimental trials. This is especially useful in the case of HDV, since animal models for this system are very limited.

Finally, our modeling effort has also highlighted some of the challenges facing any proposed treatment of HDV. The persistence of HDV under lamivudine treatment points out the necessity of not only preventing viral production, but also engineering the removal of infected cell populations capable of homeostatic population maintenance.

\section{Acknowledgements}

This work of Aaron M. Packer and Yang Kuang is supported in part by NSF DMS-0436341 and DMS-0920744. Aaron M. Packer is also supported by an Achievement Rewards for College Scientists (ARCS) scholarship.

\section{References}

[1] H. Wedemeyer, Hepatitis D revival, Liver International 31 (2011) 140.

[2] J. Taylor, Hepatitis delta virus, Virology 344 (2006) 71.

[3] M. Schaper, F. Rodriguez-Frias, R. Jardi, D. Tabernero, M. Homs, G. Ruiz, J. Quer R. Esteban, M. Buti, Quantitative longitudinal evaluations of hepatitis delta virus rna and hepatitis B virus DNA shows a dynamic, complex replicative profile in chronic hepatitis B and D, Journal of Hepatology 52 (2010) 658.

[4] R. Jardi, F. Rodriguez, M. Buti, X. Costa, M. Cotrina, R. Galimany, R. Esteban, J. Guardia, Role of hepatitis B, C, and D viruses in dual and triple infection: Influence of viral genotypes and hepatitis B precore and basal core promoter mutations on viral replicative interference, Hepatology 34 (2001) 404.

[5] K. Zachou, C. Yurdaydin, U. Drebber, G. Dalekos, A. Erhardt, Y. Cakaloglu, H. Degertekin, S. Gurel, S. Zeuzem, H. Bozkaya, V. Schlaphoff, H. Dienes, T. Bock, 
M.P. Manns, H. Wedemeyer, Quantitative HBsAg and HDV-RNA levels in chronic delta hepatitis, Liver International 30 (2010) 430.

[6] X.S. Zhang, J. Holt, J. Colvin, Mathematical models of host plant infection by helper-dependent virus complexes: why are helper viruses always avirulent?, Analytical and Theoretical Plant Pathology 90 (2000) 85

[7] X.S. Zhang, J. Holt, J. Colvin, Synergism between plant viruses: a mathematical analysis of the epidemiological implications, Plant Pathology 50 (2001) 732.

[8] B.C. de Sousa, C. Cunha, Development of mathematical models for the analysis of hepatitis delta virus viral dynamics, PLoS One 5 (2010) 1.

[9] D. Lau, E. Doo, Y. Park, D. Kleiner, P. Schmid, M. Kuhns, J. Hoofnagle, Lamivudine for chronic delta hepatitis, Hepatology 30 (1999) 546.

[10] C. Yurdaydin, H. Bozkaya, F.O. nder, et al., Treatment of chronic delta hepatitis with lamivudine vs lamivudine + interferon vs interferon, Journal of Viral Hepatitis 15 (2008) 314-321.

[11] G. Niro, A. Ciancio, H. Tillman, M. Lagget, A. Olivero, F. Perri, R. Fontana, N. Little, F. Campbell, A. Smedile, M.P. Manns, A. Andriulli, M. Rizzetto, Lamivudine therapy in chronic delta hepatitis: a multicentre randomizedcontrolled pilot study, Alimentary Pharmacology and Therapeutics 22 (2005) 227.

[12] M.A. Nowak, S. Bonhoeffer, A.M. Hill, R. Boehme, H.C. Thomas, H. McDade, Viral dynamics in hepatitis B virus infection, Proceedings of the National Academy of Science USA 93 (1996) 4398.

[13] L. Min, Y. Su, Y. Kuang, Mathematical analysis of a basic virus infection model with application to HBV infection, The Rocky Mountain Journal of Mathematics 38 (2008) 1573.

[14] K. Wang, W. Wang, S. Song, Dynamics of an HBV model with diffusion and delay, Journal of Theoretical Biology 253 (2008) 36.

[15] S.M. Ciupe, R.M. Ribeiro, P.W. Nelson, G. Dusheiko, P.Z.A.S. Perelson, R.D. Man, The role of cells refractory to productive infection in acute hepatitis B viral dynamics, Proceedings of the National Academy of Science 104 (2007). 50505050.

[16] S. Eikenberry, S. Hews, J. Nagy, Y. Kuang, The dynamics of a delay model of hepatitis B virus infection with logistic hepatocyte growth, Mathematical Bioscience and Engineering 6 (2009) 283.

[17] S.A. Gourley, Y. Kuang, J.D. Nagy, Dynamics of a delay differential model of hepatitis B virus, Journal of Biological Dynamics 2 (2008) 140
[18] S. Hews, S. Eikenberry, J.D. Nagy, Y. Kuang, Rich dynamics of a hepatitis B viral infection model with logistic hepatocyte growth, Journal of Mathematical Biology 60 (2010) 573.

[19] T. Hwang, Y. Kuang, Deterministic extinction effect of parasites on host populations, Journal of Mathematical Biology 46 (2003) 17

[20] T. Hwang, Y. Kuang, Host extinction dynamics in a simple parasite-host interaction model, Mathematical Bioscience and Engineering 2 (2005) 743.

[21] G. Farrell, N. Teoh, Management of chronic hepatitis B virus infection: a new era of disease control, Internal Medicine Journal 36 (2006) 100.

[22] E. Manesis, E. Hadziyannis, O.P. Angelopoulou, S. Hadziyannis, Prediction of treatment-related HBsAg loss in HBeAg-negative chronic hepatitis B: a clue from serum HBsAg levels, Antiviral Therapy 12 (2007) 73.

[23] L. Wolters, A.V. Nunen, P. Honkoop, A. Vossen, H. Niesters, P. Zondervan, R.D. Man, Lamivudinehigh dose interferon combination therapy for chronic hepatitis B patients co-infected with the hepatitis D virus, Journal of Viral Hepatology 7 (2000). 428-428.

[24] S.M.C. An, R.M. Ribeiro, P.W. Nelson, A.S. Perelson, Modeling the mechanisms of acute hepatitis B virus infection, Journal of Theoretical Biology 247 (2007) 23-35.

[25] H.J. Kwun, K.L. Jang, Natural variants of hepatitis B virus X protein have differential effects on the expression of cyclin-dependent kinase inhibitor p21 gene, Nucleic Acids Research 32 (2004) 2202.

[26] B. Wu, C. Li, H. Chen, J. Chang, K. Jeng, C. Chou, M. Hsu, T. Tsai, Blocking of G1/S transition and cell death in the regenerating liver of hepatitis B virus X protein transgenic mice, Biochemical and Biophysical Research Communications 340 (2006) 916.

[27] Z. Dong, J. Zhang, R. Sun, H. Wei, Z. Tian, Impairment of liver regeneration correlates with activated hepatic NKT cells in HBV transgenic mice, Hepatology 45 (2007) 1400.

[28] A.J. Hodgson, V.V. Keasler, B.L. Slagle, Premature cell cycle entry induced by hepatitis $\mathrm{B}$ virus regulatory $\mathrm{HBx}$ protein during compensatory liver regeneration, Cancer Research 68 (2008) 10341-10348.

[29] J. Beck, M. Nassal, Hepatitis B virus replication, World Journal of Gastroenterology 13 (2007) 48. 